\title{
Electrical control at the interface
}

\author{
The electric field in a Schottky barrier can be used to control spin-orbit magnetic fields.
}

\section{Daichi Chiba}

1 he efficient manipulation of magnetization is a key requirement for writing information in magnetic storage devices. One such approach is to use spin-transfer torque $(\mathrm{STT})^{1}$. Here, a spin-polarized current is passed through a ferromagnetic thin film that has a different magnetization direction to the spin axis of the injected spin-polarized current. This creates a torque that can be used to switch the magnetization in the film. Non-volatile random-access memories based on STT have already been commercially developed. This type of memory has a recording element that consists of two ferromagnetic layers separated by an insulating tunnel barrier: one layer acts as the spin-polarized current source and the other records information by STT. In addition to STT, it has been shown that current-induced spin-orbit torques (SOTs), which are the result of spin-orbit coupling, can also be used to manipulate magnetization, even in a structure with a single ferromagnetic film ${ }^{2-5}$. These current-induced effects are represented by what are known as effective spin-orbit magnetic fields. These effects have been studied in ferromagnetic metal/ non-magnetic metal bilayers at room temperature ${ }^{3,4}$ and are expected to add high-speed performance and reliability to magnetic memories. The incorporation of electrical gating into such systems could potentially enhance the efficiency of SOT devices further, because the use of an electric field to control SOT would, in principle, consume power only for charging or discharging a capacitor. However, electrical control of SOTs is challenging, mainly because the short screening length of metals makes it difficult for electric fields to penetrate bulk metal or metallic interfaces.

As well as ferromagnetic metal/nonmagnetic metal bilayer systems, currentinduced spin-orbit magnetic fields can also arise in structures that lack inversion symmetry ${ }^{2,5}$. This is the result of either Dresselhaus spin-orbit interaction, which is due to bulk inversion asymmetry of crystals, or Bychkov-Rashba spin-orbit interaction, which is due to structure inversion asymmetry. The latter mechanism is known to be more sensitive to the application of external electric fields ${ }^{6}$ and has been observed in a ferromagnetic metal/ non-magnetic semiconductor interface composed of Fe/GaAs (ref. ${ }^{5}$ ). Thus, such an interfacial structure could offer the possibility of electrical control of SOTs. Writing in Nature Electronics, Christian Back and colleagues at the University of Regensburg now show that current-induced spin-orbit magnetic fields at the interface between single-crystalline $\mathrm{Fe}$ and n-doped GaAs can be controlled by an electric field ${ }^{7}$. The electric field across this Fe/n-GaAs Schottky barrier (Fig. 1a) is tuned by applying a gate voltage at the junction (Fig. 1b), which results in modulation of the interfacial spin-orbit magnetic field.

The researchers probed the electric field dependence of the spin-orbit magnetic fields in 4-nm-thick Fe stripes on the $\mathrm{GaAs}(001)$ surface at room temperature by using a ferromagnetic resonance technique. They found that, as expected, the effective Dresselhaus and Rashba spin-orbit magnetic fields are linearly proportional to the internal electric field at the Schottky interface, and the Rashba field is more sensitive to the electric field. The relevant measurements were carried out using the Fe stripes orientated along the four main in-plane crystal directions ([100], [110], [010] and [110]), demonstrating the angular dependence of the total spinorbit field vectors and confirming that the application of the electric field can modulate the spin-orbit magnetic fields.

The manipulation of magnetism by electric fields is an active field of study ${ }^{8}$. In such work, a top-gate is typically used to apply an electric field and to modulate the carrier density. The possibility of electric control of interfacial spin-orbit fields using a conventional top-gating structure has been discussed previously ${ }^{9}$. However, with those structures, an ultrathin ferromagnetic metal, typically below $1 \mathrm{~nm}$, would be required to create a sizeable modulation, owing to the screening effect in metals. The approach of Back and colleagues is different and is not limited by the thickness of the ferromagnetic metal. Thus, the use of such Schottky barriers for controlling spin-orbit

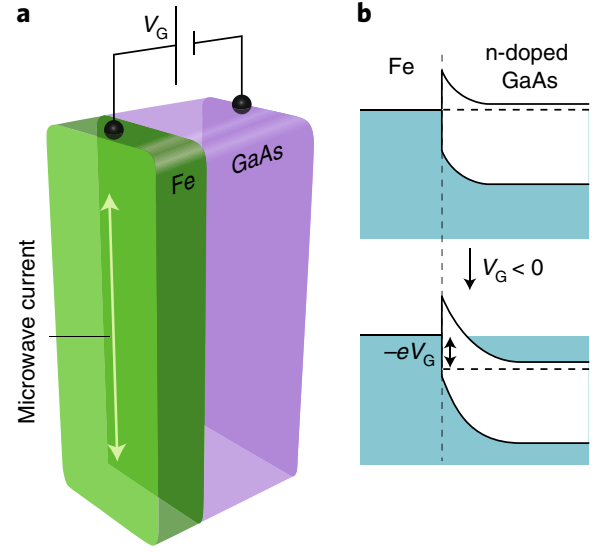

Fig. 1 | Electric field control of spin-orbit magnetic fields in a Fe/n-GaAs Schottky junction. a, Simplified schematic of the device structure and the experimental set-up. A microwave current is applied to generate time-dependent interfacial spin-orbit magnetic fields at the $\mathrm{Fe} / \mathrm{GaAs}$ (001) Schottky interface. A ferromagnetic resonance technique is used to detect those fields, which can be modified by application of a gate voltage $\left(V_{G}\right)$. b, Schematic band diagrams around the Schottky junction. Application of the gate voltage changes the interfacial electric field, which is proportional to the interfacial spin-orbit interaction.

magnetic fields is an attractive alternative for developing low-power SOT devices.

\section{Daichi Chiba}

Department of Applied Physics, The University of Tokyo, Tokyo, Japan.

e-mail:dchiba@ap.t.u-tokyo.ac.jp

Published online: 13 June 2018

https://doi.org/10.1038/s41928-018-0090-4

\footnotetext{
References

1. Brataass, A., Kent, A. D. \& Ohno, H. Nat. Mater. 11, 372-381 (2012).

2. Chernyshov, A. et al. Nat. Phys. 5, 656-659 (2009).

3. Miron, I. M. et al. Nature 476, 189-193 (2011).

4. Liu, L. Q. et al. Science 336, 555-558 (2012).

5. Chen, L. et al. Nat. Commun. 7, 13802 (2016).

6. Nitta, J., Akazaki, T. \& Takayanagi, H. Phys. Rev. Lett. 78 1335-1338 (1997).

7. Chen, L. et al. Nat. Electron. https://doi.org/10.1038/s41928-0180085-1 (2018).

8. Matsukura, F., Tokura, Y. \& Ohno, H. Nat. Nanotech. 10, 209-220 (2015).

9. Liu, R. H. et al. Phys. Rev. B 89, 220409(R) (2014).
} 